\title{
EVALUACIÓN CLÍNICA DE PARAGONIMIOSIS EN GATOS INFECTADOS EXPERIMENTALMENTE CON Paragonimus mexicanus
}

\section{Clinical Evaluation of Paragonimiosis in Cats Experimentally Infected WITH Paragonimus mexicanus}

\author{
Christina Gallardo V. ${ }^{1}$, Eva Casas A. ${ }^{1,5}$, Alina Huiza F. ${ }^{4}$, Raúl Sevilla A. ${ }^{4}$, \\ Diego Díaz C. ${ }^{2}$, Joana Ramos G. ${ }^{1}$, Fidel Suárez A. ${ }^{3}$
}

\section{ResUmen}

El objetivo del presente estudio fue describir signos clínicos asociados con la infección experimental con Paragonimus mexicanus en gatos. Se emplearon ocho gatos de 2.5 a 4 meses de edad, que fueron infectados con 7 a 20 metacercarias por vía oral. En el examen físico durante la fase aguda se encontró aumento de la temperatura corporal, cuadros de tos productiva y un aumento de tamaño del nódulo linfático inguinal, y en la fase crónica cuadros de tos productiva, reflejo traqueal positivo y aumento del murmullo vesicular. En el hemograma se encontró leucocitosis con grados variables de linfocitosis, neutrofilia y eosinofilia en la fase aguda, y leucocitosis por aumento de eosinófilos en la fase crónica. A través de la ecografía abdominal se encontró engrosamiento de la pared vesicular, esplenomegalia, hepatomegalia y aumento de ecogenicidad hepática, especialmente durante la fase aguda. Los hallazgos radiológicos tempranos incluyeron focos de bronquiectasia y de engrosamiento bronquial y aumento de radiopacidad de lóbulos pulmonares, y en etapas posteriores se observaron estructuras radiopacas ovoides en parénquima pulmonar y bronquiectasia. El periodo entre la inoculación de las metacercarias hasta la observación de huevos de $P$. mexicanus en heces fue de 41 a 70 días. En la necropsia se confirmó la presencia de quistes y parásitos a nivel pulmonar.

Palabras clave: Paragonimiosis, Paragonimus mexicanus, Paragonimus peruvianus, gato, signos clínicos, fase aguda, fase crónica

${ }^{1}$ Laboratorio de Microbiología y Parasitología Veterinaria, ${ }^{2}$ Clínica de Animales Menores, ${ }^{3}$ Laboratorio de Medicina Veterinaria Preventiva, Facultad de Medicina Veterinaria, Universidad Nacional Mayor de San Marcos, Lima

${ }^{4}$ Instituto de Medicina Tropical «Daniel Alcides Carrión», Facultad de Medicina Humana, Universidad Nacional Mayor de San Marcos, Lima

${ }^{5}$ E-mail: evacasas99@gmail.com

Recibido: 8 de abril de 2012

Aceptado para publicación: 30 de noviembre de 2013 
The objective of this study was to describe the clinical signs associated to the experimental infection of Paragonimus mexicanus in cats. Eight cats of 2.5 to 4 months of age were orally infected with 7 to 20 metacercariae. In the physical examination during the acute phase was found fever, wet cough, and enlargement of the inguinal lymph node, while in the chronic phase wet cough, positive tracheal reflex and increase of the breath sound. On the complete blood count was found leukocytosis with different degrees of lymphocytosis, neutrophilia and eosinophilia in the acute phase and leukocytosis with an increase of eosinophilia in the chronic phase. On the ultrasonography examination, enlargement of the vesicular wall, hepatomegaly, splenomegaly and increase in liver echogenicity were found, especially during the acute phase. The early radiological findings included bronchiectasis focus, bronchial thickening and increased radiopacity of lung lobes, while in the chronic phase radiopaque ovoid structures in the pulmonary parenchyma and bronchiectasis were observed. The period between infection and presence of $P$. mexicanus eggs in the faeces was between 41 and 71 days. The necropsy confirmed the presence of cysts and parasites in the lungs.

Key words: Paragonimiosis, Paragonimus mexicanus, Paragonimus peruvianus, cat, clinical signs, acute phase, chronic phase

\section{INTRODUCCIÓN}

La paragonimiosis es una zoonosis parasitaria causada por tremátodos del género Paragonimus, siendo Paragonimus mexicanus $(=P$. peruvianus) la especie notificada como principal agente en el Perú (Alvarado et al., 2004). Su presencia fue reportada por primera vez en el país en 1910 (Barton, 1910), al verificarse la presencia de huevos de Paragonimus spp en el esputo de un poblador de Trujillo, considerándose al agente causal como Paragonimus westerman hasta 1967, por ser la especie más conocida y estudiada a nivel mundial (Ibáñez y Jara, 1992).

En 1967 se aísla, por primera vez en el país, metacercarias y parásitos pulmonares del género Paragonimus (Ibáñez y Miranda, 1967), y que más tarde fueron confirmados como una especie nueva para la ciencia, denominándose $P$. peruvianus (Ibáñez y Jara, 1992); sin embargo, años más tarde se demostró que $P$. peruvianus era similar a $P$. mexicanus (Huiza, 2004).
Los hospederos definitivos de $P$. mexicanus suelen ser el hombre, que se infecta de manera accidental y animales domésticos como el perro y gato, y diversos mamíferos silvestres pequeños o medianos como el hurón, zarigüeya y pecarí. Además, requiere de dos invertebrados como hospederos intermediarios en su ciclo evolutivo. En el Perú han sido descritos el caracol del género Aroapyrgus como el primer hospedero intermediario y cangrejos del género Hypolobocera como segundo hospedero intermediario (Atías y Náquira, 1997).

La infección de los hospederos definitivos, entre ellos el hombre, ocurre al ingerir cangrejos de río, crudos o mal cocidos, que se encuentran infestados con metacercarias de P. mexicanus (Cornejo et al., 2000). Luego de la ingestión, las metacercarias migran a través del peritoneo y el diafragma hasta llegar al pulmón y, ocasionalmente, a otros órganos, donde se alojan y se transforman en adultos. Estos, mediante reproducción sexual producen huevos, que salen con el esputo y son expulsados por la boca o las heces (Uruburu et al., 2008). 
Las metacercarias de $P$. westermani penetran a partir de la mucosa intestinal, a diferencia de $P$. mexicanus que inicia su migración a nivel de esófago y estómago (Tantaleán et al., 1974). Las metacercarias de P. mexicanus carecen, asimismo, de membrana quística (Rojas, 2003), razón por la cual inician la migración tan pronto como ingresan al tubo digestivo.

Debido a las diferencias biológicas entre $P$. mexicanus y $P$. westermani, la sintomatología clínica no es la misma. La paragonimiosis por $P$. westermani se manifiesta durante las tres primeras semanas de la ingestión de las metacercarias enquistadas con diarrea, dolor abdominal, fiebre, urticaria, hepatoesplenomegalia y eosinofilia. Estos síntomas se presentan durante la migración de los tremátodos desde la cavidad abdominal hacia los pulmones. La fase crónica (infección pulmonar) se manifiesta con tos persistente, hemoptisis, dolor torácico, pérdida de peso, y con menos frecuencia con disnea y fiebre (Moyou et al., 2003).

En la paragonimiosis causada por $P$. mexicanus, el periodo invasivo o fase aguda de la infección suele pasar desapercibido y la concurrencia del paciente a un Centro de Salud suele hacerse cuando tiene tos persistente o cuando la expectoración es sanguinolenta (Atías y Náquira, 1997), confundiéndose con la tuberculosis (Cornejo et al., 1999).

El diagnóstico en humanos se realiza a través de exámenes parasitológicos, radiológicos y serología. En el hemograma se observa leucocitosis con eosinofilia importante (23-57\%) (Uribarren, 2009). Los hallazgos radiológicos incluyen derrame pleural, neumotórax, bronquiectasias y fibrosis pulmonar (Mukae et al., 2001). El gato doméstico, hospedero definitivo de $P$. mexicanus, es utilizado en infecciones experimentales (Huiza et al., 2001), dado que es posible recuperar ejemplares adultos del tejido pulmonar en un 30-95\% de los casos (Tantaleán y Huiza, 1986).
Solo se ha descrito la sintomatología clínica de paragonimiosis en humanos, incluyendo la causada por P. mexicanus; sin embargo, el cuadro clínico causado por esos parásitos es muy variable y falta describir el cuadro clínico causado por $P$. mexicanus en animales. El objetivo del presente estudio fue describir los signos clínicos asociados con la infección experimental de $P$. mexicanus en el gato.

\section{Materiales y Métodos}

\section{Lugar de Estudio}

El manejo, cuidado y evaluación de los gatos se realizó en los ambientes del bioterio del Instituto de Medicina Tropical «Daniel Alcides Carrión» de la Facultad de Medicina Humana, Universidad Nacional Mayor de San Marcos (UNMSM), entre setiembre de 2009 a enero de 2010. Los exámenes complementarios se hicieron en la Clínica de Animales Menores y en los laboratorios de la Facultad de Medicina Veterinaria (FMV), UNMSM, y en la clínica privada Vet Center. El protocolo del estudio y manejo animal fue aprobado por el Comité de Ética y Bienestar Animal de la FMV-UNMSM.

\section{Animales}

Se emplearon ocho gatos domésticos de 2.5 a 4 meses de edad (6 hembras y dos machos) sin distinción de raza. Los animales experimentales cumplieron con los siguientes criterios antes de la infección experimental: aparente buen estado de salud, hemograma completo con valores dentro de los rangos normales, órganos abdominales sin alteración evidente en la ecografía abdominal, parénquima pulmonar sin alteración evidente en la radiografía de tórax, y sin evidencia de formas parasitarias en heces en el examen coproparasitológico.

Los gatos fueron alojados en jaulas individuales de 70 x 50 x $40 \mathrm{~cm}$ (largo $\mathrm{x}$ ancho $\mathrm{x}$ alto) en un ambiente con servicios de agua 
y desagüe. El ambiente y las jaulas fueron aseados y desinfectados diariamente con hipoclorito de sodio al $5.25 \%$. Además, se contó con un pediluvio con desinfectante en base a cresoles en la entrada del ambiente.

\section{Control Sanitario}

Los gatos fueron desparasitados externamente con Fipronil en dosis de $15 \mathrm{mg} / \mathrm{kg}$ de peso vivo, e internamente con una combinación comercial a base de Pirantel Pamoato $(35 \mathrm{mg} / \mathrm{ml})$ y Praziquantel $(25 \mathrm{mg} / \mathrm{ml})$ en dosis de $1 \mathrm{ml} / 5 \mathrm{~kg}$ de peso vivo, vía oral, por tres días consecutivos y con una dosis única 10 días después. La desparasitación se hizo entre 60 a 70 días antes de la infección experimental. Además, fueron inmunizados con la Vacuna Triple Felina (rinotraqueitis viral felina, calicivirus felino y panleucopenia felina) a los 2.5 y 3 meses de edad, 10 días después de la última desparasitación.

Los animales fueron pesados y se realizó una evaluación clínica completa antes y después de la infección experimental. También se realizaron exámenes complementarios para verificar el estado de salud pre y post-inoculación (hemograma completo, examen coproparasitológico, ecografía abdominal y radiología de tórax).

\section{Metacercarias}

Las metacercarias de $P$. mexicanus se obtuvieron del hepatopáncreas de cangrejos vivos del género Hypolobocera procedentes del departamento de Cajamarca. Se extrajo el hepatopáncreas, se colocó en una placa Petri y se le extendió con ayuda de un estilete. Se buscaron las metacercarias con ayuda de un estereoscopio, se retiraron con una pipeta Pasteur, se colocaron en frascos pequeños con solución salina, y se mantuvieron en refrigeración por 72 horas hasta la inoculación.

Previo a la inoculación se confirmó la viabilidad de las metacercarias por observa- ción directa al microscopio (100\% de viabilidad a las 48 y $80 \%$ a las $72 \mathrm{~h}$ ). Se inocularon de 7 a 20 metacercarias viables por gato, vía oral, con ayuda de una pipeta Pasteur con punta roma, depositando el inóculo en la faringe del animal.

\section{Metodología Experimental}

Las evaluaciones y exámenes clínicos realizados se muestran en la Fig. 1.

\section{Necropsia}

Los animales fueron sacrificados nueve semanas (días 66, 67, 74 y 85) después de la infección experimental. Los animales ayunaron por $12 \mathrm{~h}$ previo a la eutanasia. Para el sacrificio, se inyectó Promazil IM en dosis de $1 \mathrm{mg} / \mathrm{kg}$ como tranquilizante y una dosis letal de pentobarbital sódico IV de $40 \mathrm{mg} / \mathrm{kg}$.

En la necropsia se confirmó la presencia de quistes y parásitos a nivel pulmonar. Se evaluaron las lesiones macroscópicas en órganos de la cavidad abdominal y torácica. Se colectaron muestras de pulmón, colon, recto y nódulos linfáticos mediastínicos, mesentérico yeyunal y cecal; además de tejido inflamatorio del mediastino. No obstante, los resultados histopatológicos no forman parte del presente estudio.

\section{Análisis de Datos}

El estudio es de carácter descriptivo y los resultados se presentan mediante frecuencias absolutas y relativas, a través de cuadros y figuras (fotografías).

\section{Resultados}

Los gatos fueron positivos a $P$. mexicanus entre los días 41 a 50 de la infección, según la técnica de sedimentación, con excepción de un gato que fue positivo a los 70 días de la infección. 


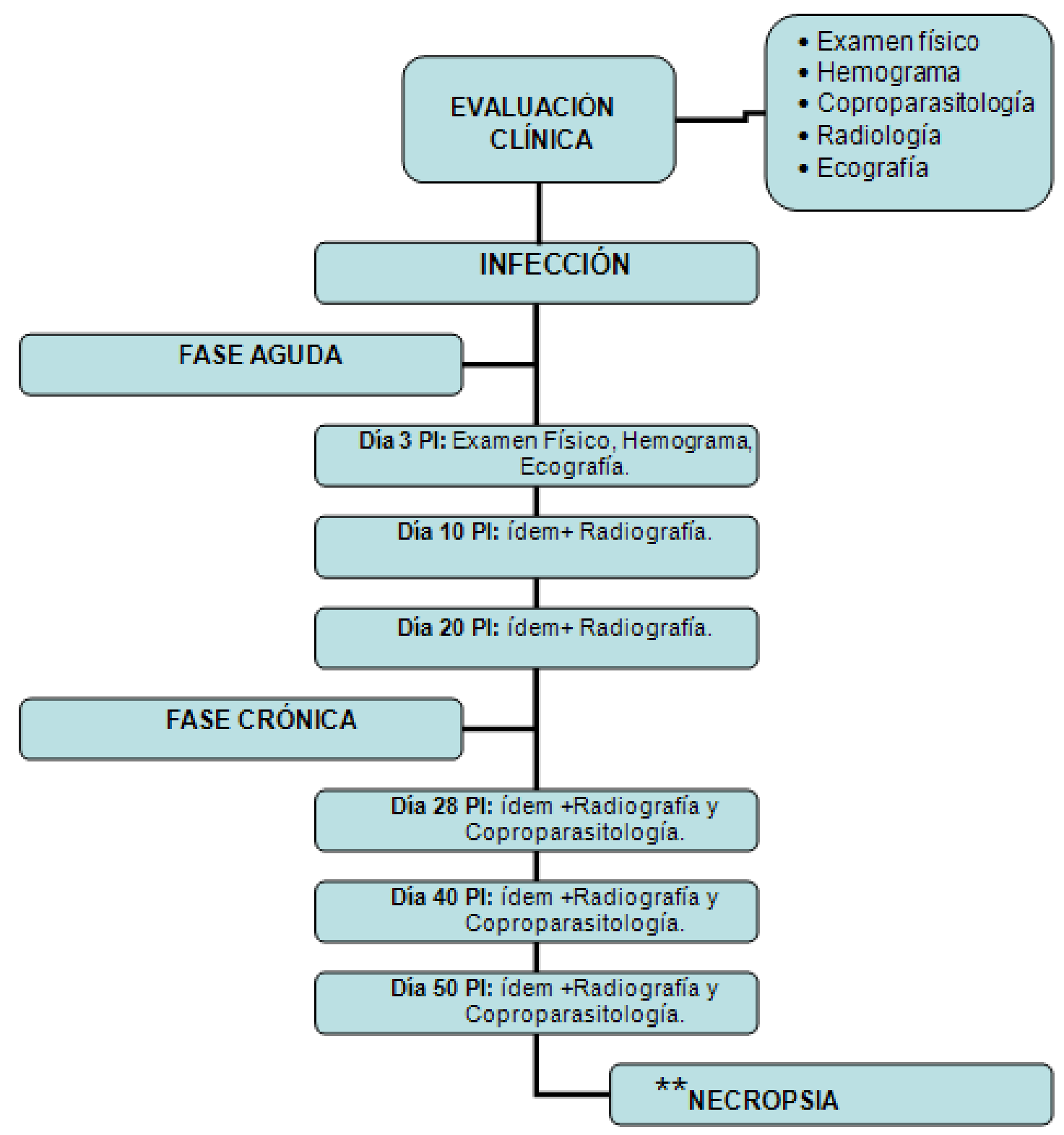

Figura 1. Evaluaciones y exámenes clínicos realizados en ocho gatos experimentalmente infectados con metacercarias vivas de Paragonimus mexicanus. **Se tomaron placas radiográficas de tórax un día antes de la necropsia. PI: post-inoculación; Ídem: examen físico, hemograma y ecografía 
Cuadro 1. Frecuencia de signos clínicos (n) encontrados al examen físico durante la fase aguda (1-20 días) y crónica (>20 días) de paragonimiosis en ocho gatos infectados experimentalmente con metacercarias de Paragonimus mexicanus (Lima, 2009)

\begin{tabular}{lcccccccc}
\hline \multirow{2}{*}{ Signos clínicos } & \multicolumn{3}{c}{ Fase aguda } & \multicolumn{5}{c}{ Fase crónica } \\
\cline { 2 - 8 } & $1-3$ & $4-10$ & $11-20$ & $21-28$ & $29-40$ & $41-50$ & $>50$ \\
\hline $\begin{array}{l}\text { Aumento de temp. corporal } \\
\text { Aumento del nódulo }\end{array}$ & 2 & 1 & - & - & - & - & - \\
$\begin{array}{l}\text { linfático inguinal } \\
\text { Tos productiva esporádica }\end{array}$ & - & - & - & 1 & 1 & 1 & - \\
Tos productiva persistente & - & - & - & 1 & 3 & 3 & 1 \\
$\begin{array}{l}\text { Reflejo traqueal (+) } \\
\text { Aumento del murmullo }\end{array}$ & - & - & - & - & 1 & 5 & 8 \\
vesicular & - & - & - & - & 1 & 5 & 8 \\
$\begin{array}{l}\text { Estertores húmedos a la } \\
\text { auscultación }\end{array}$ & - & - & - & - & - & - & 1 \\
\hline
\end{tabular}

Cuadro 2. Frecuencia de hallazgos ecográficos (n) durante la evaluación clínica de la fase aguda (1-20 días) y crónica (>20 días) de paragonimiosis en ocho gatos infectados experimentalmente con metacercarias de Paragonimus mexicanus (Lima, 2009)

\begin{tabular}{|c|c|c|c|c|c|c|}
\hline \multirow{2}{*}{ Hallazgos ecográficos } & \multicolumn{3}{|c|}{ Fase aguda } & \multicolumn{3}{|c|}{ Fase crónica } \\
\hline & 3 & 10 & 20 & 28 & $38-40$ & $48-50$ \\
\hline $\begin{array}{l}\text { Aumento de ecogenicidad en } \\
\text { peritoneo }\end{array}$ & 1 & - & - & - & - & - \\
\hline Esplenomegalia & 1 & 4 & 3 & 2 & 2 & 2 \\
\hline Hepatomegalia & 1 & 5 & 2 & 2 & 1 & 1 \\
\hline Aumento de ecogenicidad en hígado & 1 & 1 & 1 & 1 & - & - \\
\hline $\begin{array}{l}\text { Engrosamiento de la pared vesicular } \\
(1-3.7 \mathrm{~mm})\end{array}$ & 2 & 4 & 7 & 5 & 6 & 4 \\
\hline $\begin{array}{l}\text { Aumento de tamaño del nódulo } \\
\text { linfático inguinal derecho }\end{array}$ & - & - & - & 1 & 1 & - \\
\hline $\begin{array}{l}\text { Nódulos linfáticos mesentéricos } \\
\text { aumentados de tamaño }\end{array}$ & - & - & - & - & 1 & 2 \\
\hline $\begin{array}{l}\text { Banda hiperec ogénica entre corteza } \\
\text { y médula renal }\end{array}$ & - & 3 & 1 & 2 & 1 & 2 \\
\hline
\end{tabular}


Cuadro 3. Frecuencia de hallazgos radiológicos (n) durante la evaluación clínica de la fase aguda (1-20 días) y crónica ( $>20$ días) de paragonimiosis en ocho gatos infectados experimentalmente con metacercarias de Paragonimus mexicanus (Lima, 2009)

\begin{tabular}{|c|c|c|c|c|c|c|}
\hline \multirow{2}{*}{ Hallazgos radiológicos } & \multicolumn{2}{|c|}{ Fase aguda } & \multicolumn{4}{|c|}{ Fase crónica } \\
\hline & 10 & 20 & 28 & $38-40$ & $48-50$ & Nec. ${ }^{1}$ \\
\hline $\begin{array}{l}\text { Focos de bronquiectasia a nivel del } 6^{\circ} \\
\text { al } 10^{\circ} \text { espacio intercostal }\end{array}$ & 3 & 3 & - & - & 3 & - \\
\hline $\begin{array}{l}\text { Focos de engrosamiento bronquial a } \\
\text { nivel de } 6^{\circ} \text { al } 12^{\circ} \text { espacio intercostal }\end{array}$ & 2 & 4 & 5 & 7 & 5 & 2 \\
\hline $\begin{array}{l}\text { Puntos radiopacos a nivel del } 4^{\circ} \text { al } 12^{\circ} \\
\text { espacio intercostal }\end{array}$ & 2 & 2 & 2 & 2 & 1 & - \\
\hline $\begin{array}{l}\text { Aumento de radiopacidad del lóbulo } \\
\text { apical }\end{array}$ & - & 2 & 1 & 5 & 2 & 2 \\
\hline $\begin{array}{l}\text { Aumento de radiopacidad del lóbulo } \\
\text { diafragmático }\end{array}$ & 1 & 2 & 2 & 4 & 3 & 1 \\
\hline $\begin{array}{l}\text { Aumento de radiopacidad a nivel del } \\
\text { lóbulo cardiaco }\end{array}$ & - & - & 1 & 2 & 1 & - \\
\hline $\begin{array}{l}\text { Estructura radiopaca de forma ovoide } \\
\text { en parénquima pulmonar }\end{array}$ & - & - & - & 2 & 3 & 1 \\
\hline $\begin{array}{l}\text { Estructura con halo radiopaco y centro } \\
\text { radiolúcido en parénquima pulmonar }\end{array}$ & - & - & - & - & 1 & 2 \\
\hline $\begin{array}{l}\text { Infiltrado no definido en parénquima } \\
\text { pulmonar }\end{array}$ & - & 2 & 1 & - & 1 & 6 \\
\hline
\end{tabular}

El cuadro clínico en la fase aguda de la enfermedad se caracterizó por un aumento de la temperatura corporal, cuadros de tos productiva y un aumento de tamaño del nódulo linfático inguinal. En la fase crónica se evidenciaron clínicamente los cuadros de tos productiva en todos los animales, incluyendo tos persistente, reflejo traqueal positivo y aumento del murmullo vesicular. El gato inoculado con más metacercarias $(n=20)$ llegó incluso a presentar estertores húmedos (crepitaciones) a la auscultación (Cuadro 1).

Los hemogramas mostraron en la fase aguda de la enfermedad cuadros de leucocitosis con grados variables de linfo- citosis, neutrofilia y eosinofilia, aumentando considerablemente hacia el día 20 post-inoculación. En la fase crónica de la enfermedad, los niveles de linfocitos y eosinófilos alcanzaron su mayor nivel en el día 28 postinoculación.

En la ecografía abdominal se encontró el peritoneo e hígado con aumento ecogénico, esplenomegalia, hepatomegalia, engrosamiento de la pared vesicular y banda hiperecogénica entre corteza y médula renal, especialmente durante la fase aguda de la enfermedad. Además, en la fase crónica hubo aumento de tamaño del nódulo linfático inguinal derecho y de los nódulos linfáticos mesentéricos (Cuadro 2). 
Los hallazgos en las radiografías de tórax durante la fase aguda de la enfermedad fueron focos de bronquiectasia y de engrosamiento bronquial, puntos radiopacos y aumento de radiopacidad en los lóbulos pulmonares, especialmente en la fase crónica de la enfermedad (Cuadro 3).

En la necropsia, los nódulos linfáticos mesentéricos regionales a nivel de yeyuno (Fig. 2A), ciego, colon y recto (Fig. 2B) estaban aumentados de tamaño. Además, había gran cantidad de formaciones redondas de 2 a $3 \mathrm{~mm}$ de diámetro, levemente protruidos y de color blanquecino, distribuidas de manera difusa, a nivel del colon y recto (Figs. 2C, 2D) en la mayoría de los gatos.

En el examen de la cavidad torácica, a nivel de glotis, tráquea y bronquios, se observó moderada presencia de exudado mucoso marrón-ocre de consistencia viscosa (Fig. 3A). También se observaron estructuras quísticas globosas marrón-ocre en la pleura mediastínica y en el parénquima pulmonar, de 1 a $3 \mathrm{~cm}$ de diámetro (Figs. 3B, 3C), que

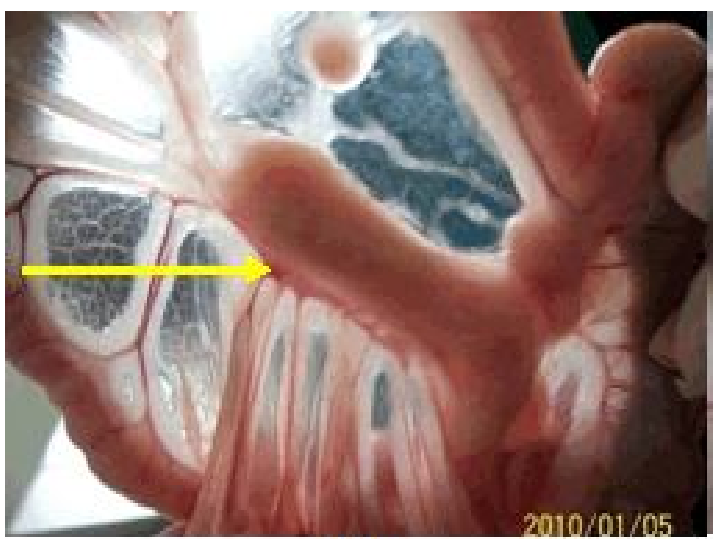

(A)

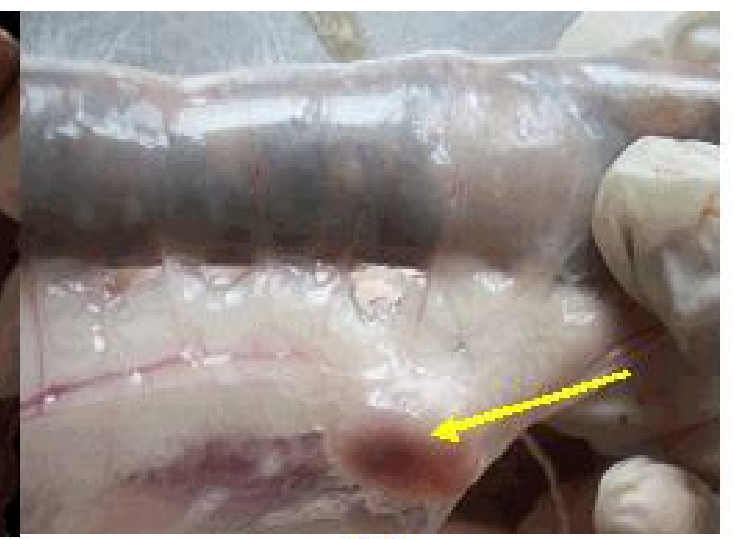

(B)

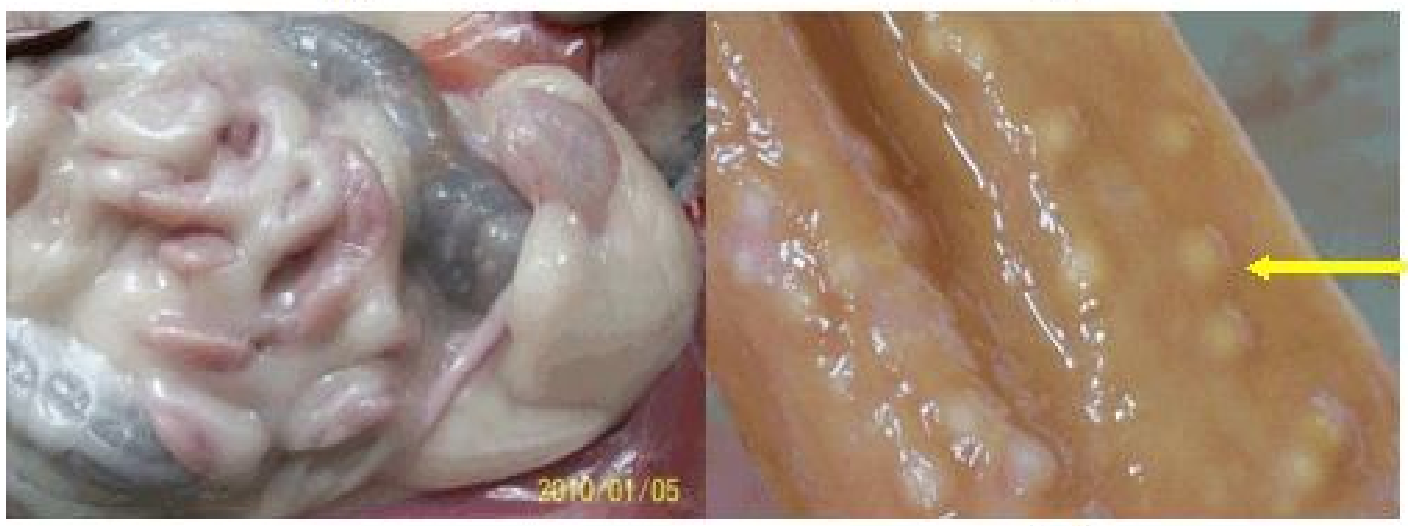

(C)

Figura 2. Hallazgos en la cavidad abdominal en ocho gatos experimentalmente inoculados con metacercarias de Paragonimus mexicanus (Lima, 2009). (A) Nódulo linfático mesentérico yeyunal aumentado de tamaño; (B) Nódulo linfático mesentérico en recto aumentado de tamaño; (C) Pequeñas formaciones redondas de color blanquecino a nivel del colon; (D) Formaciones redondas de color blanquecino en la mucosa de colon y recto 


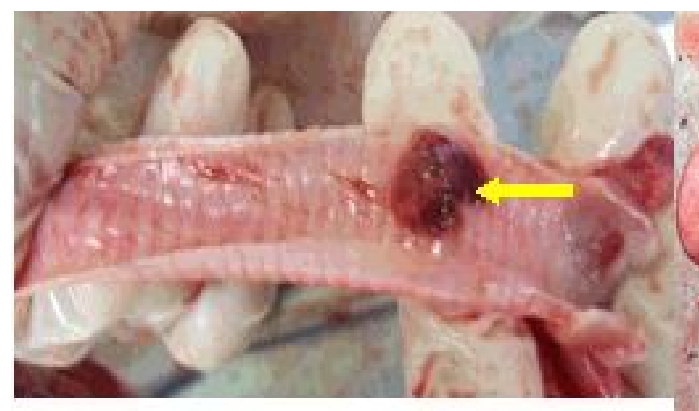

(A)

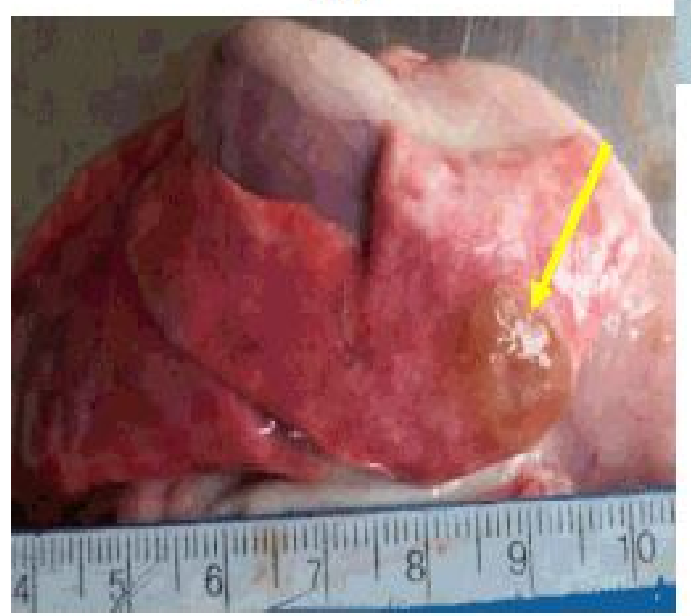

(C)

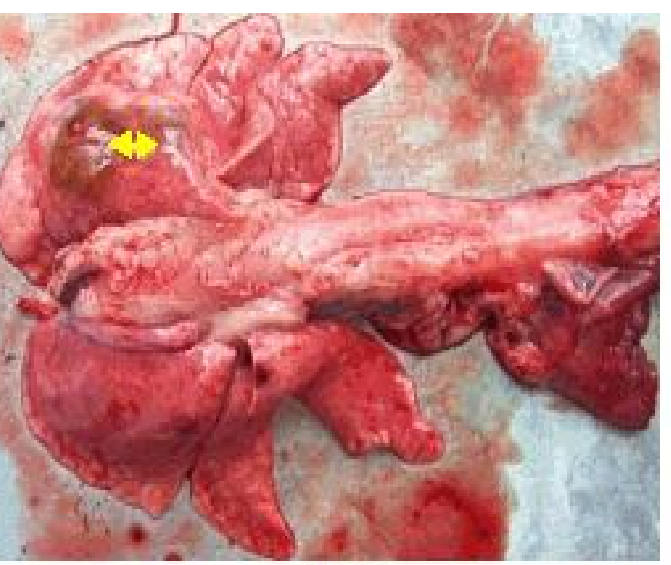

(B)

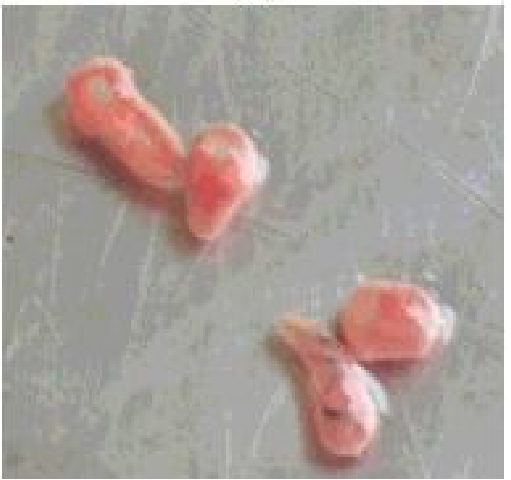

(D)

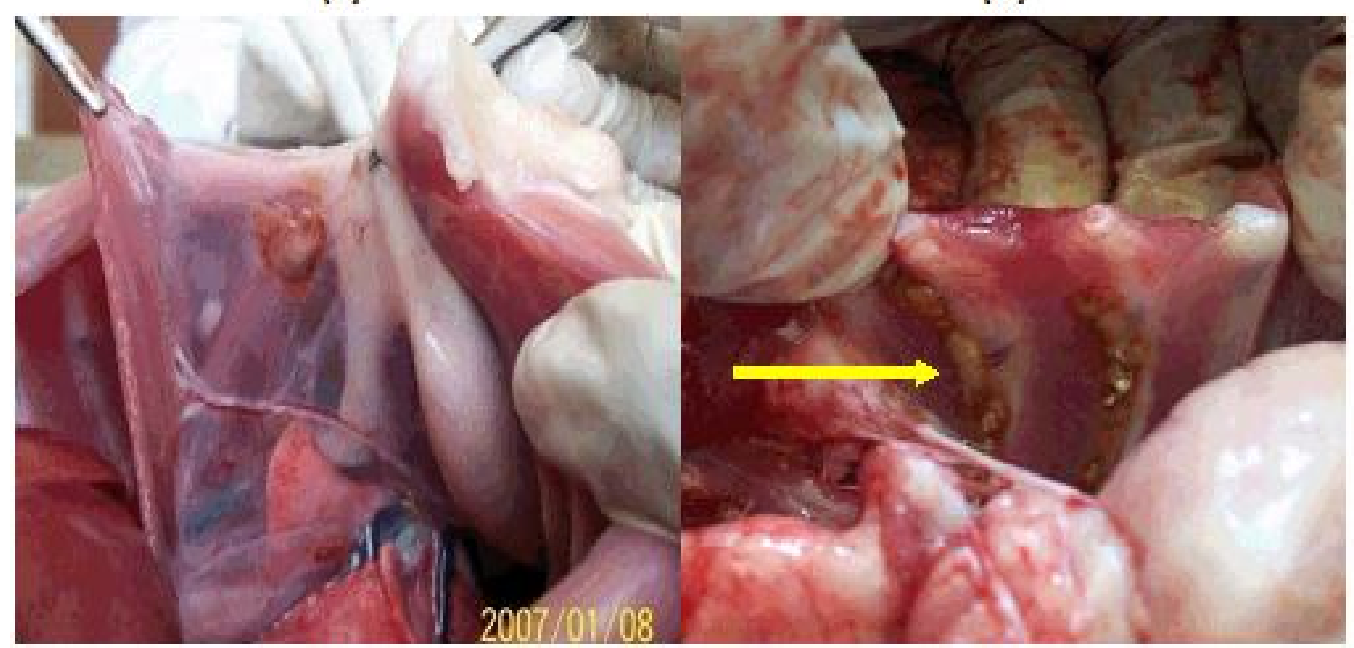

(E)

(F)

Figura 3. Hallazgos en la cavidad torácica en ocho gatos experimentalmente inoculados con metacercarias de Paragonimus mexicanus (Lima, 2009). (A) Presencia de exudado mucoso viscoso en tráquea; (B) Dos estructuras redondeadas 3 × $2 \mathrm{~cm}$ de diámetro en el lóbulo diafragmático; (C) Estructura redondeada globosa de $1 \mathrm{~cm}$ de diámetro en parénquima del lóbulo apical; (D) Estructuras movibles en el parénquima pulmonar, en forma de pera de 4-8 $\mathrm{mm}$ de largo x 2-4 $\mathrm{mm}$ de ancho, sugerentes a formas parasitarias adultas de P. mexicanus; (E) Pleura mediastínica con tejido de infiltración; (F) Formaciones redondeadas, superficiales y duras al tacto a nivel de los espacios intercostales 
al corte fluyó líquido marrón, observándose la presencia de estructuras movibles en forma de pera, de color castaño rosa de 4 a 8 $\mathrm{mm}$ de largo x 2 a $4 \mathrm{~mm}$ de ancho, con un aplanamiento en sentido dorsoventral sugerente a formas parasitarias adultas de $P$. mexicanus (Fig. 3D).

En la pleura mediastínica se observó un área de tejido de infiltración de color marrón ocre, sobre todo en la zona que se adhiere al esternón y diafragma (Fig. 3E), así como formaciones redondeadas y superficiales de color nácar y dura al tacto a nivel de los espacios intercostales (Fig. 3F) y en la superficie pulmonar. Los nódulos linfáticos mediastínicos estaban aumentados de tamaño.

Todos los gatos resultaron infectados con estadios adultos de $P$. mexicanus entre los 65 y 85 días posteriores a la infección. Los quistes característicos, de color pardo, de 1 a $3 \mathrm{~cm}$ de diámetro se encontraron en los pulmones de los ocho gatos. Los quistes estaban distribuidos a nivel de lóbulo apical, accesorio y diafragmático. Una de las formas quísticas tuvo una ubicación extrapulmonar.

Los quistes parasitarios encontrados a nivel pulmonar fueron compatibles con las descripciones radiográficas tomadas un día antes de la necropsia, la cual los indicó como infiltrados no definidos en parénquima pulmonar.

\section{Discusión}

Los resultados obtenidos permitieron describir la presencia de signos clínicos en fase aguda y crónica de la paragonimiosis en gatos.

\section{Hallazgos en el examen clínico}

Durante la fase aguda, que coincide con la migración de los parásitos desde el tubo digestivo hacia los pulmones, se pudo observar un aumento de la temperatura en el $25 \%$ de los gatos, aunque solo uno de ellos pre- sentó temperatura febril hasta el día 8. Este periodo febril ocurre usualmente en el 10-20\% de los pacientes humanos al principio del proceso de la enfermedad (Procop, 2009), y es uno de los signos clínicos menos observados (Jeon et al., 2005). El aumento de temperatura puede atribuirse a los pirógenos liberados por las metacercarias durante su migración larvaria, tal como ocurre con migraciones larvarias viscerales de otros parásitos (Palmieri, 2005).

El aumento de tamaño del nódulo linfático inguinal derecho, detectado al examen clínico y ecográfico durante 15 días pudo deberse a una migración errática de Paragonimus (Tantaleán y Huiza, 1986; Uribarren, 2009). El parásito, como parte de su migración dentro del hospedero definitivo, necesita permanecer un tiempo en los músculos abdominales (Tantaleán y Huiza, 1986), lo cual ha sido corroborado por los trabajos con tomografía computarizada (CT) (Lee et al., 2007).

Las larvas de $P$. mexicanus causan lesiones parenquimatosas intrapulmonares debido a que se establecen adyacentes a los conductos aéreos pequeños, formando los quistes parasitarios (Jeon et al., 2005). Este proceso de migración causa lesiones mecánicas en los bronquios contribuyendo al desarrollo de focos de bronquiectasia (Im et al., 1992), causando tos persistente y exceso de flema, como ha sido observado en el presente estudio (Cuadro 1).

La bronquitis puede pasar desapercibida en gatos en reposo y presentar auscultación pulmonar normal (Ettinger y Feldman, 2002), hasta que comience a evidenciarse cuadros de tos productiva persistente. La intensidad del murmullo vesicular puede estar incrementada alrededor de una zona pulmonar alterada, debido a una reacción compensadora funcional de las partes sanas del pulmón (Montoya, 2006), condición que se presentó en el animal con mayor cantidad de metacercarias inoculadas. En animales con tos húmeda o productiva suele auscultarse crepitaciones en la zona de proyección de los pulmones (Montoya, 2006). 


\section{Hallazgos en el hemograma}

Los exámenes de sangre periférica permiten la detección de leucocitosis y eosinofilia en muchos pacientes humanos con paragonimiosis pulmonar (Jeon et al., 2005). En el presente estudio, el $87.5 \%$ de los gatos inoculados presentaron grados variables de leucocitosis y linfocitosis, $25 \%$ de neutrofilia y el $100 \%$ de eosinofilia.

La leucocitosis observada el día 3 de la inoculación en tres gatos pudo estar asociada a la inmunoestimulación causada por la migración de las larvas de $P$. mexicanus. Por otro lado, el aumento de linfocitos parece ser secundario a la inmunoestimulación por los microorganismos persistentes, aunque estos incrementos tienden a ser modestos (Ettinger y Feldman, 2002). En los gatos con elevado nivel de leucocitos en el día 10 de la inoculación se observó, además, una mayor predominancia de linfocitos y neutrófilos, lo cual se correlaciona con la penetración y migración de larvas en órganos abdominales, causando la hepatomegalia y esplenomegalia observada en el estudio.

En el día 20 de la inoculación se observó leucocitosis con predominancia de eosinófilos en el $65 \%$ de los animales. Las enfermedades parasitarias con extenso contacto tisular tienden a estar asociadas con eosinofilia (Ettinger y Feldman, 2002; Rebar et al., 2002). La eosinofilia asociada con infecciones parasitarias es dependiente de las células $\mathrm{T}$ y están mediadas por factores solubles que incluyen interleucina-3, en especial interleucina-5 y GM-CSF (Factor de estimulación de colonias de granulocitosmacrófagos), razón por la cual se observan casos de eosinofilia asociada a linfocitosis; sin embargo, hay pocos estudios que han examinado la relación entre la interleucina-5 y la paragonimiosis (Mukae et al., 2001).

Los casos de esosinofilia aumentaron durante la fase crónica, llegando a niveles máximos en el día 28 de la inoculación. Posteriormente, el número de animales con esosinofilia disminuyó aumentando los casos de leucocitosis, los cuales podrían deberse a los focos inflamatorios en pulmones. Al parecer, la eosinofilia se presenta solamente cuando los parásitos están vivos y migrando. Es así que aunque la eosinofilia es un parámetro importante en la sospecha de paragonimiosis en las etapas tempranas de la infección, una carencia de eosinofilia no necesariamente descarta la posibilidad de paragonimiosis (Jeon et al., 2005).

\section{Hallazgos ecográficos}

Las alteraciones en órganos abdominales, probablemente causadas por la migración larvaria de $P$. mexicanus (Tantaleán y Huiza, 1986), comenzaron a ser visibles al examen ecográfico en el día 10 post-inoculación; principalmente casos de hepatomegalia $(62.5 \%$ de los animales), engrosamiento de la pared vesicular (50\%), esplenomegalia (50\%) y presencia de bandas hiperecogénicas entre la corteza y médula renal (25\%).

En el día 20 post-inoculación comenzaron a disminuir las alteraciones ecográficas en los órganos abdominales, probablemente debido a que la migración larvaria ya no ocurre a nivel de estos órganos. No obstante, los casos de engrosamiento de la pared vesicular aumentaron $(87.5 \%)$, pero sin evidencia de signos clínicos específicos de una colecistitis; posiblemente por el paso de las larvas del parásito a ese nivel. Esta ruta migratoria no ha sido reportada en el gato, pero se tiene historia de un caso de colecistitis crónica calculosa en un paciente de un hospital de Lima, Perú (Salinas et al., 1999). En animales menores, los parásitos de la vesícula biliar o conductos biliares rara vez son identificados, pero se reporta que el trematodo Platynosomum fastosum se localiza permanentemente en el conducto biliar en forma asintomática o causando fibrosis u obstrucción (Ettinger y Feldman, 2002).

Debido al alto número de casos de hepatomegalia $(87 \%)$ y engrosamiento de la pared vesicular $(100 \%)$, podría especularse 
que la larva de $P$. mexicanus migra por la cavidad abdominal, dirigiéndose primero al parénquima hepático o a la vesícula biliar como parte de su migración en cavidad abdominal, antes de dirigirse a los músculos abdominales y seguir su migración hacia los pulmones. Se sabe que la migración larvaria a órganos o músculos en el hospedero definitivo varía según la especie de Paragonimus y de hospedero (Tantaleán y Huiza, 1986).

\section{Hallazgos radiológicos}

La larva de $P$. mexicanus se le puede encontrar en los pulmones a partir del día 12 de la inoculación (Tantaleán y Huiza, 1986). Las larvas maduran en tremátodos adultos dentro del pulmón y se rodean de formaciones quísticas. La apariencia de las lesiones pulmonares en las radiografías varía con la etapa de la infección y la reacción del tejido que lo rodea (Im et al., 1992).

En el día 10 se observó como parte del proceso migratorio, focos de bronquiectasia (37.5\%) y de engrosamiento bronquial (25\%), puntos radiopacos a nivel de espacios intercostales $(25 \%)$ y un caso de radiopacidad del lóbulo diafragmático. Los puntos radiopacos en parénquima pulmonar se pueden asociar a lesiones durante el proceso de penetración pleural de las larvas. La efusión pleural o neumotórax puede presentarse en casos de ingestión de gran cantidad de metacercarias (Im et al., 1992). En este estudio, se trabajó con una dosis moderada de metacercarias, razón por la cual no se presentaron estos casos.

Luego de la penetración pleural, los parásitos juveniles se unen y establecen adyacentes a los bronquios y bronquiolos, formando quistes parasitarios que pueden causar una prolongada inflamación bronquial que contribuye al desarrollo de bronquiectasia (Jeon et $a l ., 2005)$, tal y como lo observado en el presente estudio.

En el día 20 post-inoculación se evidenció un aumento en el número de casos de focos de engrosamiento bronquial, el cual se incrementó hasta $62.5 \%$ en el día 28 y a $87.5 \%$ en el día 38-40, debido a la migración activa de la larva. Además, comienzan a aumentar las áreas pulmonares radiopacas. Uno de los hallazgos radiológicos iniciales de la paragonimiosis es la consolidación irregular del espacio aéreo, debido a una neumonía hemorrágica causada por el parásito migrante (Im et al., 1992), lo cual se traduce como pequeños infiltrados pulmonares.

Durante el proceso de maduración de los parásitos juveniles en adultos se van formando quistes a su alrededor (quistes parasitarios), debido al infarto por la obstrucción de una arteriola o una vena por el parásito y la expansión del pequeño espacio aéreo por un parásito intraluminal. Cuando el espacio quístico se llena con fluido hemorrágico y se rodea por consolidación periquística del espacio aéreo, aparece como una consolidación localizada, tipo masa, en las radiografías. Estos quistes comenzaron a observarse el día 38-40 post-inoculación; asimismo, los quistes que se comunican con el bronquiolo adyacente aparecen como quistes aéreos dentro del pulmón consolidado o como sombras anulares (Im et al., 1992).

En el día 50 de la inoculación se pudo observar gran cantidad de focos de bronquiectasia, ocupando uno de ellos un gran espacio, causado probablemente por parásitos localizados en el área peribronquial. Así, la obstrucción del conducto aéreo adyacente resulta en atelectasia (Im et al., 1992). Asimismo, estos autores reportaron que el caso de conexión del quiste parasitario con el bronquio, aparece aire o gas dentro del quiste en la CT.

Radiológicamente, la paragonimiosis pulmonar puede manifestarse en un hospedero desde una radiografía torácica absolutamente normal hasta infiltrados difusos con hidroneumotórax bilateral. Esto es dependiente de la etapa de desarrollo de la enfermedad (Jeon et al., 2005). La frecuencia de las lesiones parenquimatosas intrapulmonares en 
el presente estudio fue alta, y los infiltrados no definidos en parénquima pulmonar a nivel de los últimos espacios intercostales fueron las lesiones parenquimatosas más comunes (100\%).

\section{Hallazgos en la necropsia}

El porcentaje de recuperación de parásitos a nivel pulmonar varió entre 20 y $100 \%$, similares a los porcentajes reportados por Tantaleán y Huiza (1986) en gatos pequeños.

\section{Conclusiones}

- En la fase aguda de la paragonimiosis se observó temperatura febril y tos productiva, mientras que en la fase crónica se observaron evidentes signos clínicos respiratorios (tos productiva, reflejo traqueal positivo, aumento del murmullo vesicular).

- Se encontró leucocitosis debida principalmente a eosinofilia y linfocitosis.

- A través de la ecografía abdominal se encontró engrosamiento de la pared vesicular, esplenomegalia, hepatomegalia y aumento de ecogenicidad hepática, especialmente durante la fase aguda.

- Los hallazgos radiológicos tempranos incluyeron focos de bronquiectasia y de engrosamiento bronquial y aumento de radiopacidad de lóbulos pulmonares, y en etapas posteriores se observaron estructuras radiopacas ovoides en parénquima pulmonar y bronquiectasia.

- El periodo entre la inoculación de las metacercarias hasta la observación de huevos de $P$. mexicanus fue de 41 a 70 días.

- La necropsia confirmó la presencia de quistes y parásitos a nivel pulmonar.

\section{Literatura Citada}

1. Alvarado P, Pariona L, Beltrán M. 2004. Casos de paragonimiasis (paragonimiosis) en el Hospital Nacional Sergio
E. Bernales (Lima, Perú). Rev Perú Med Exp Salud Pública 21: 107-110.

2. Atías A, Náquira C. 1997. Parasitología médica. Lima: Public Técnica Mediterráneo. $615 \mathrm{p}$.

3. Cornejo W, Náquira C, Espinoza $F$, Huiza A, Sevilla C. 1999. La paragonimiasis en escolares del valle de Condebamba, Cajamarca-Perú. Anales Fac Med 60: 196-198.

4. Cornejo W, Huiza A, Espinoza F, Alva P, Sevilla C, Centurión W. 2000. Paragonimosis in the Cajabamba and Condebamba districts, Cajamarca, Peru. Rev Inst Med Trop S Paulo 42: 245-247.

5. Ettinger S, Feldman E. 2002. Tratado de medicina interna veterinaria. Vol 2. Buenos Aires: Ed Inter-Médica. 2116 p.

6. Huiza A, Sevilla C, Espinoza Y. 2001. Infección experimental del hámster (Mesocricetus auratus) con metacercarias de Paragonimus mexicanus (peruvianus). Rev Med Exp 18: 3-4.

7. Ibáñez N, Jara C. 1992. Paragonimus peruvianus: Metacercarias en nuevo hospedador intermediario Hypolobocera chilensis: de la provincia de San Miguel, Cajamarca, Perú. Rev Peru Med Trop 6: 63-70.

8. Ibáñez N, Miranda H. 1967. Paragonimiasis: hallazgo de formas adultas de Paragonimus sp en Felis domesticus y en Didelphys sp (hurón). En: IV Congreso Latinoamericano de Microbiología. Lima.

9. Im Jung-Gi, Whang Hee Young, Kim Woo Sun, Han Man Chung, Shim Young-Soo, Cho Seung-Yull. 1992. Pleuropulmonary paragonimiasis: Radiologic findings in 71 patients. Am J Roentgenol 159: 39-43.

10. Jeon Kyeongman, Jung Koh Won, Kim Hojoong, Jung Kwon O, Sung Kim Tae, Soo Lee Kyung, Han Joungho. 2005. Clinical features of recently diagnosed pulmonary paragonimiasis in Korea. Chest 128: 1423-1430.

11. Lee CH, Im JG, Goo JM, Lee HJ, Hong ST, Shen CH, Chung DH, et al. 2007. Serial $C T$ findings of Paragonimus 
infested dogs and the Micro-CT findings of the worm cysts. Korean J Radiol 8: 372-381.

12. Montoya AA. 2006. Enfermedades respiratorias en pequeños animales. Buenos Aires: Ed Inter-Médica. 655 p.

13. Moyou S, Kefie A, Dreyfuss D. 2003. An epidemiological study of pleuropulmonary paragonimiasis among pupils in the peri-urban zone of Kumba town, Meme Division, Cameroon. BMC Public Health 3:40.

14. Mukae H, Taniguchi H, Matsumoto N, Iiboshi H, Ashitani J, Matsukura S, Nawa Y. 2001. Clinicoradiologic features of pleuropulmonary Paragonimus westermani on Kyusyu Island, Japan. Chest 120: 514-520.

15. Palmieri OJ. 2005. Enfermedades infecciosas. Buenos Aires: Ed HA Macchi. 780 p.

16. Procop GW. 2009. North American paragonimiasis (caused by Paragonimus kellicotti) in the context of global paragonimiasis. Clin Microbiol Rev 22: 415-446.

17. Rebar A, MacWilliams P, Metzeger F. 2002. Manual de hematología de perros y gatos. Barcelona: Ed Multimédica. 152 p.
18. Rojas M. 2003. Nosoparasitosis de perros y gatos peruanos. Lima: Martegraf. Perú. 83 p.

19. Salinas C, Gutiérrez G Casavilca S. 1999. Paragonimiasis vesicular: reporte de un caso. Rev Gastroenterol Perú 19: 57-58.

20. Tantaleán M, Huiza A. 1986. La ruta migratoria de la larva de Paragonimus mexicanus Miyazaki e Ishi, 1969. En el gato doméstico infectado experimentalmente. Rev Ciencias UNMSM 74: 63-69.

21. Tantaleán M, Náquira C, Huiza A, Delgado E. 1974. La vía de penetración de la larva de Paragonimus peruvianus en animales de experimentación. Rev Inst Med Trop Sao Paulo 16: 332-336.

22. Uribarren BT. 2009. Paragonimosis. UNAM. [Internet] Disponible en: http:// www.facmed.unam.mx/deptos/ microbiologia/parasitologia/ paragonimosis.html

23. Uruburu M, Granada M, Velásquez L. 2008. Distribución parcial de Paragonimus (Digenea: Troglotrematidae) en Antioquia, por presencia de metacercarias en cangrejos dulciacuícolas. Biomédica 28: 562-568. 